\title{
A Comprehensive Study of the X-ray Bursts from the Magnetar Candidate 1E 2259+586
}

\author{
Fotis P. Gavriil ${ }^{*}$, Victoria M. Kaspi ${ }^{* \dagger}$ and Peter M. Woods** \\ ${ }^{*}$ Department of Physics, Rutherford Physics Building, McGill University, 3600 University Street, Montreal, \\ Quebec, H3A 2T8, Canada \\ ${ }^{\dagger}$ Department of Physics and Center for Space Research, Massachusetts Institute of Technology, Cambridge, MA \\ 02139 \\ ${ }^{* *}$ Space Science Research Center, National Space Science and Technology Center, Huntsville, AL 35805, USA
}

\begin{abstract}
We present a statistical analysis of the X-ray bursts observed from the 2002 June 18 outburst of the Anomalous X-ray Pulsar (AXP) 1E 2259+586, observed with the Proportional Counter Array aboard the Rossi X-ray Timing Explorer. We show that the properties of these bursts are similar to those of Soft Gamma-Repeaters (SGRs). The similarities we find are: the burst durations follow a log-normal distribution which peaks at $99 \mathrm{~ms}$, the differential burst fluence distribution is well described by a power law of index -1.7 , the burst fluences are positively correlated with the burst durations, the distribution of waiting times is well described by a log-normal distribution of mean $47 \mathrm{~s}$, and the bursts are generally asymmetric with faster rise than fall times. However, we find several quantitative differences between the AXP and SGR bursts. Specifically, there is a correlation of burst phase with pulsed intensity, the AXP bursts we observed exhibit a wider range of durations, the correlation between burst fluence and duration is flatter than for SGRs, the observed AXP bursts are on average less energetic than observed SGR bursts, and the more energetic AXP bursts have the hardest spectra - the opposite of what is seen for SGRs. We conclude that the bursts are sufficiently similar that AXPs and SGRs can be considered united as a source class yet there are some interesting differences that may help determine what physically differentiates the two closely related manifestations of neutron stars.
\end{abstract}

\section{INTRODUCTION}

Soft gamma repeaters (SGRs) are an exotic class of Galactic sources that are now commonly accepted as being magnetars - isolated, young neutron stars that are powered by the decay of an ultra-high magnetic field. The evidence for high surface fields $\left(\sim 10^{14}-10^{15} \mathrm{G}\right)$ comes from several independent lines of reasoning [1, 2, 3, 4]. These include: the high dipolar magnetic fields implied by the spin properties of SGRs seen in quiescence under the assumption of magnetic dipole braking [5, 6]; the requirement of a magnetar-strength field to confine the energy released in the tails of hyper-Eddington outbursts seen from two SGRs [7, 8]; the requirement of a high field to allow the decay rate necessary to power the burst and persistent emission [4, 9]; and the magnetic suppression of the Thomson cross-section, which allows hyper-Eddington bursts to be observed [2]. For a review of SGRs, see Kouveliotou et al. [these proceedings].

Anomalous X-ray pulsars (AXPs), another exotic class of Galactic neutron stars, have also been suggested to be magnetars [4]. This is because of their anomalously bright X-ray emission which can be explained neither by conventional binary accretion models nor ro- tation power [10]. Also, their spin parameters, as for SGRs, imply large magnetic fields under standard assumptions of magnetic braking. They also have similar, though on average softer, X-ray spectra compared with those of SGRs in quiescence. However, unlike SGRs, in the $>20 \mathrm{yr}$ since the discovery of the first AXP [11], none was seen to exhibit SGR-like bursts. For this reason, alternative models involving unconventional accretion scenarios have been proposed to explain AXP emission [12, 13, 14]. See Kaspi et al. [these proceedings] for a review of AXPs.

The magnetar model for AXPs was recently given a boost when SGR-like bursts were detected from two AXPs. Gavriil et al. [15] reported on the discovery of two $\mathrm{X}$-ray bursts in observations obtained in the direction of AXP 1E 1048.1-5937. The temporal and spectral properties of those bursts were similar only to those seen only in SGRs. However, the AXP could not be definitely identified as the burster. On 2002 June 18, a major outburst was detected unambiguously from AXP 1E 2259+586, involving over 80 bursts as well as significant spectral and timing changes in the persistent emission [16]. Those bursts demonstrated that AXPs are capable of exhibiting behavior observed, until now, uniquely in SGRs, there- 


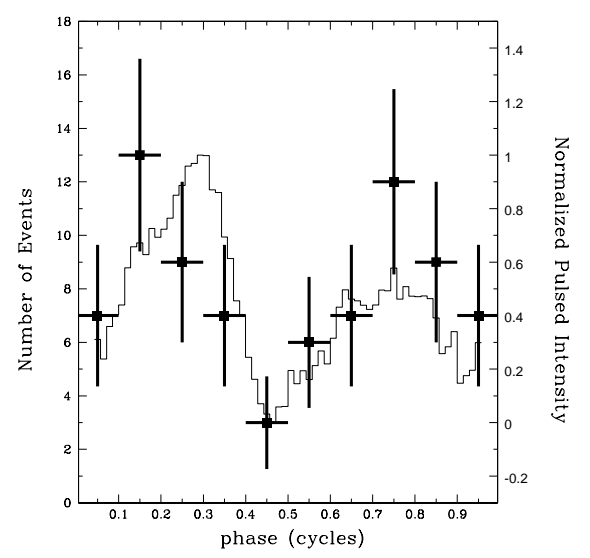

FIGURE 1. Distribution of the pulse phases of 1E 2259+586 which correspond to the times of the burst peaks (solid points). The solid curve is the folded 2-60 keV light curve of the 2002 June 18 observation with the bursts omitted.

fore implying a clear connection between the two source classes. Such a connection was predicted only by the magnetar model [4].

\section{RESULTS}

The results presented here were obtained using the Proportional Counter Array [PCA; 17] on board the Rossi $X$-ray Timing Explorer (RXTE). We use RXTE to monitor all five known AXPs on a regular basis as part of a long-term monitoring campaign [see 18 , and references therein]. On 2002 June 18, during one of our regular monitoring observations, the AXP 1E 2259+586 exhibited an SGR-like outburst [16]. The bursting behavior was detected by online $R X T E$ monitors during the observation. The observation spanned three orbits and had total on-source integration time $10.7 \mathrm{ks}$. Following the outburst, Target of Opportunity observations of the source were initiated the next day and continued at different intervals over the subsequent weeks, however no more bursts were seen.

Our burst searching algorithm returned 80 significant bursts from the 2002 June 18 observation. Most bursts were single-peaked and had durations $\lesssim 1$ s. A small handful $(\sim 12)$ were bright and had clear fast-rise, exponential decay morphology. Four bursts were multipeaked. Some bursts $(\sim 5 \%)$ were approximately symmetric, a few $(\sim 3 \%)$ fell faster than they rose while most fell slower than they rose.

We calculated the occurance in pulse phase for each burst using the time of the burst peak and the rotational ephemeris given by Kaspi et al. [16]. Comparing the burst phase distribution to the pulse profile of

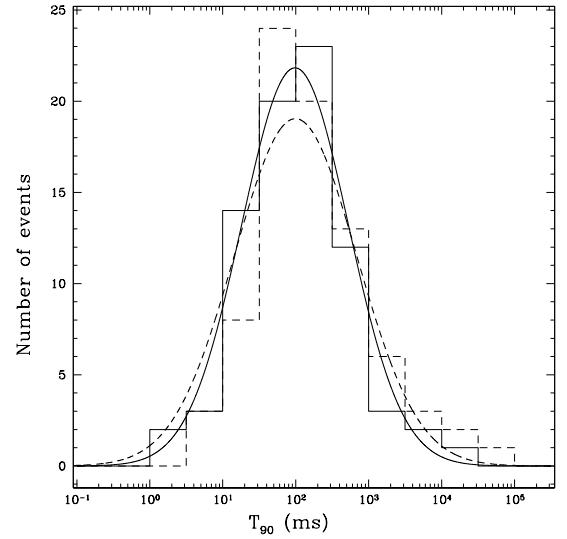

FIGURE 2. Distribution of $\mathrm{T}_{90}$ durations for the bursts observed from 1E 2259+586. The solid histogram line shows the observed binned distribution, while the dashed histogram line shows the corrected distribution (see text). The solid curve represents the best-fit log-normal model for the observed data, while the dashed curve is the best-fit log-normal model for the corrected data.

$1 \mathrm{E} 2259+586$ at the time of the outburst, a correlation is seen (Fig.11, where most of the bursts tend to occur when the pulsed intensity is high. We note that the two bursts seen from the AXP 1E 1048.1-5937 [15] were also coincident with the pulse peak, which strengthens the argument that 1E 1048.1-5937 was the source of those bursts.

The $\mathrm{T}_{90}$ duration is the time between when $5 \%$ and $95 \%$ of the total background-subtracted burst counts have been accumulated [e.g. 19]. SGR T 90 distributions follow a log-normal distribution whose mean and standard deviation vary with source [e.g. 19]. At first we fit the measured values of $\mathrm{T}_{90}$ for the $1 \mathrm{E} 2259+586$ bursts with this model and found it to characterize the distribution well (Fig. 2). For low signal-to-noise bursts, $\mathrm{T}_{90}$ can be substantially underestimated. We corrected for this problem via Monte Carlo simulations [see 20]. The corrected $\mathrm{T}_{90}$ distribution is shown in Figure 2 The best-fit mean is $99.31 \mathrm{~ms}$ with a range of $14.4-683.9 \mathrm{~ms}$ for one standard deviation.

We measured the fluence of each burst and then grouped them in equispaced logarithmic bins. The distribution of burst fluences is displayed in Figure 3 The low-end fluences are underrepresented because of sensitivity drop-off. Excluding the points having fluence $\lesssim 20$ PCA counts, the distribution is well modeled by a simple power law. Using least-squares fitting we find a bestfit power-law index of $-0.7 \pm 0.1$, which corresponds to a differential spectrum $\mathrm{d} N / \mathrm{d} F \propto F^{-1.7 \pm 0.1}$. The fluences in the $2-60 \mathrm{keV}$ band range from $\sim 5 \times 10^{-11}$ to $\sim 7 \times 10^{-9} \mathrm{erg} \mathrm{cm}^{-2}$. These imply burst energies in the range $\sim 5 \times 10^{34}$ to $\sim 7 \times 10^{36} \mathrm{erg}$, assuming isotropic 


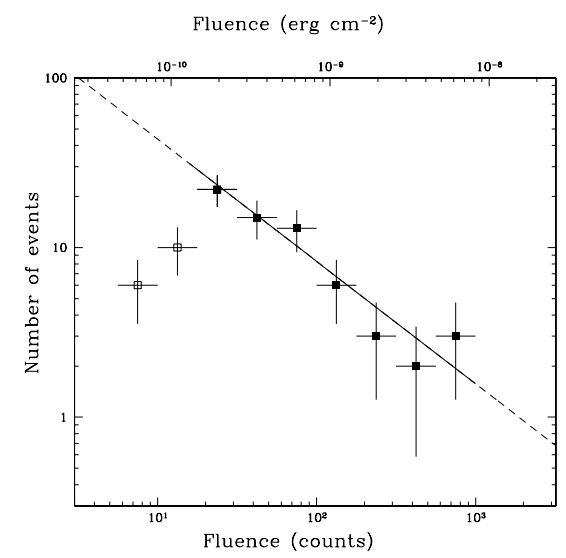

FIGURE 3. Distribution of the $2-60 \mathrm{keV}$ fluence for each burst. Solid points represent average values of fluence in equispaced logarithmic bins for which our observations had full sensitivity. The open points suffered from reduced sensitivity. The best-fit line was determined using the solid points only and is shown as a solid line; the dashed lines are its extrapolation.

emission and a distance of $3 \mathrm{kpc}$ to the source [21]. The sum total of all burst fluences is $5.6 \times 10^{-8} \mathrm{erg} \mathrm{cm}^{-2}$, corresponding to energy $6.0 \times 10^{37} \mathrm{erg}(2-60 \mathrm{keV})$.

Göğüş et al. [19] also find a clear correlation between burst durations and total burst fluence. A similar correlation is seen in our 1E $2259+586$ data. To quantify it, we grouped the $\mathrm{T}_{90}$ values in equispaced logarithmic bins and determined group-averaged fluences for each bin. Least-squares fitting to a simple power-law model yields $F \propto \mathrm{T}_{90}^{+0.54 \pm 0.08}$, with reduced $\chi^{2}=1.0$.

We measured the peak flux in a $61.25-\mathrm{ms}$ time bin for each burst. Our burst-identifying algorithm is less sensitive to bursts of smaller peak flux, we compensated for this effect via simulations [see 20]. Using least-squares fitting we found that the corrected distribution is well modelled by a simple power law with index $-1.42 \pm 0.13$. Peak fluxes in a 61.25 -ms time bin range from $\sim 1 \times 10^{-9}$ to $\sim 1 \times 10^{-7} \mathrm{erg} \mathrm{cm}^{-2} \mathrm{~s}^{-1}$, which imply peak luminosities in the range $\sim 1 \times 10^{36}$ to $\sim 1 \times 10^{38} \mathrm{erg} \mathrm{s}^{-1}$. On shorter time scales we find 5 bursts with peak fluxes which are super-Eddington. The peak fluxes in a 1/2048 s time bin for these bursts range from $\sim 2 \times 10^{38}$ to $\sim 8 \times 10^{38} \mathrm{erg} \mathrm{s}^{-1}$.

Burst rise and fall time distributions were well modelled by log-normal distributions. For the rise time distribution, we find a mean of $2.43 \mathrm{~ms}$ and a range of $0.51-$ $11.51 \mathrm{~ms}$ for one standard deviation. For the fall time distribution, we find mean $13.21 \mathrm{~ms}$ and a range of 3.52$49.55 \mathrm{~ms}$ for one standard deviation.

SGR waiting times, defined as the temporal separations of adjacent bursts, are found to follow log-normal distributions [22, 23]. We measured the waiting time for

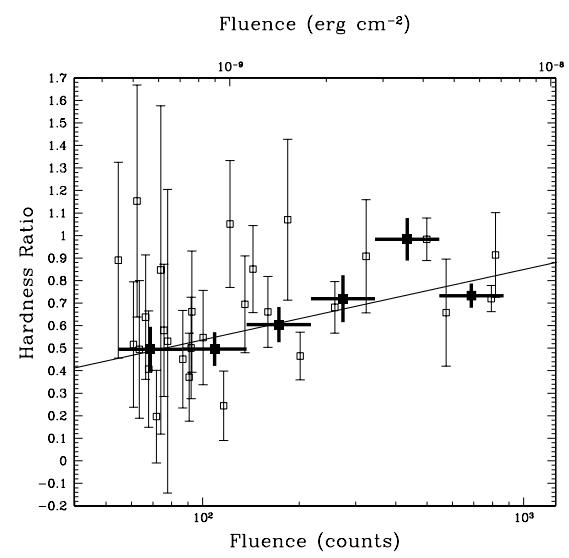

FIGURE 4. Hardness ratio $(H)$ versus fluence $(F)$. The open points are hardness ratio measurements for individual bursts. The solid points are weighted averages of hardness ratios for bursts in equispaced logarithmic fluence bins. The line represents the best-fit logarithmic function for the weighted averages, $H=0.31 \times \log F-0.09$.

the $1 \mathrm{E} 2259+586$ events, excluding those interrupted by Earth occultations. Our waiting time distribution is also well modelled by a log-normal distribution with mean $46.7 \mathrm{~s}$ and a range of 10.5-208.4 $\mathrm{s}$ for one standard deviation. We find no correlation between the burst energy, duration and the waiting time until the next burst, nor with the elapsed time since the previous burst. There is a correlation between the waiting time and the event time, which implies that the mean of our waiting time distribution depends on the time at which we started observing the outburst.

Göğüş et al. [19] noted that SGR bursts tend to soften with increasing burst energy. We studied the hardness ratio/fluence relationship by extracting spectra and creating response matrices separately for each burst. Hardness ratios were defined as the ratio of the counts in the 10$60 \mathrm{keV}$ band to those in the $2-10 \mathrm{keV}$ band as in Göğüs et al. [19]. Also following Göğüs et al. [19], we divided the bursts into equispaced logarithmic fluence bins and calculated a weighted average hardness ratio for each bin. Figure 4 shows the weighted mean hardness ratios as a function of fluence. A clear positive correlation is seen.

\section{DISCUSSION}

The bursts we have observed for 1E 2259+586 are clearly similar to those seen uniquely in SGRs. As concluded by Gavriil et al. [15] and Kaspi et al. [16], AXPs and SGRs clearly share a common nature, as has been predicted by the magnetar model. The bursts seen in the 2002 June 
18 outburst of $1 \mathrm{E} 2259+586$ are qualitatively similar to those seen in SGRs, and in many ways quantitatively similar. Specifically:

- the mean burst durations are similar

- the differential burst fluence spectrum is well described by a power law of index -1.7 , similar to those seen in SGRs (and earthquakes and solar flares)

- burst fluences are positively correlated with burst durations

- the distribution of and mean waiting times are similar

- the burst morphologies are generally asymmetric, with rise times usually shorter than burst durations

However, there are some interesting quantitative differences between the properties of the AXP and SGR bursts. These may help shed light on the physical difference(s) between these classes. The differences can be summarized as:

- there is a significant correlation of burst phase with pulsed intensity, unlike in SGRs

- the AXP bursts have a wider range of burst duration (though this may be partly due to different analyses procedures)

- the correlation of burst fluence with duration is flatter for AXPs than it is for SGRs (although when selection effects are considered, this correlation should really be seen as an upper envelope for AXPs and SGRs)

- the fluences for the AXP bursts are generally smaller than are in observed SGR bursts

- the more energetic AXP bursts have the hardest spectra, whereas for SGR bursts, they have the softest spectra

- under reasonable assumptions, SGRs undergo outbursts much more frequently than do AXPs

Given the rarity of AXP bursts coupled with the unique information that detection of such bursts provides, observing more outbursts is obviously desirable. Continued monitoring is thus clearly warranted, and $R X T E$ with its large area and flexible scheduling is the obvious instrument of choice.

\section{ACKNOWLEDGMENTS}

We are grateful to C. Kouveliotou, M. Lyutikov, S. Ransom, M.S.E. Roberts, D. Smith, and C. Thompson for useful discussions. This work was supported in part by NSERC, NATEQ, CIAR and NASA. This research has made use of data obtained through the High Energy Astrophysics Science Archive Research Center Online Service, provided by the NASA/Goddard Space Flight Center.

\section{REFERENCES}

1. Duncan, R. C., and Thompson, C., ApJ, 392, L9-L13 (1992).

2. Paczyński, B., Acta Astron., 42, 145-153 (1992).

3. Thompson, C., and Duncan, R. C., MNRAS, 275, 255-300 (1995).

4. Thompson, C., and Duncan, R. C., ApJ, 473, 322-342 (1996).

5. Kouveliotou, C., Dieters, S., Strohmayer, T., van Paradijs, J., Fishman, G. J., Meegan, C. A., Hurley, K., Kommers, J., Smith, I., Frail, D., and Murakami, T., Nature, 393, 235-237 (1998).

6. Kouveliotou, C., Strohmayer, T., Hurley, K., Van Paradijs, J., Finger, M. H., Dieters, S., Woods, P., Thompson, C., and Duncan, R. C., ApJ, 510, L115-L118 (1999).

7. Mazets, E. P., Golenetskii, S. V., Il'inskii, V. N., Apetkar', R. L., and Gur'yan, Y. A., Nature, 282, 587 (1979).

8. Hurley, K., Cline, T., Mazets, E., Barthelmy, S., Butterworth, P., Marshall, F., Palmer, D., Aptekar, R., Golenetskii, S., Ill'Inskii, V., Frederiks, D., McTiernan, J., Gold, R., and Trombka, T., Nature, 397, 41-43 (1999).

9. Goldreich, P., and Reisenegger, A., ApJ, 395, 250-258 (1992).

10. Mereghetti, S., and Stella, L., ApJ, 442, L17-L20 (1995).

11. Fahlman, G. G., and Gregory, P. C., Nature, 293, 202-204 (1981).

12. van Paradijs, J., Taam, R. E., and van den Heuvel, E. P. J., $A \& A, 299, \mathrm{~L} 41-\mathrm{L} 44$ (1995).

13. Chatterjee, P., Hernquist, L., and Narayan, R., ApJ, 534, 373-379 (2000).

14. Alpar, M. A., ApJ, 554, 1245-1254 (2001).

15. Gavriil, F. P., Kaspi, V. M., and Woods, P. M., Nature, 419, 142-144 (2002).

16. Kaspi, V. M., Gavriil, F. P., Woods, P. M., Jensen, J. B., Roberts, M. S. E., and Chakrabarty, D., ApJ, 588, L93 (2003).

17. Jahoda, K., Swank, J. H., Giles, A. B., Stark, M. J., Strohmayer, T., Zhang, W., and Morgan, E. H., Proc. SPIE, 2808, 59 (1996).

18. Gavriil, F. P., and Kaspi, V. M., ApJ, 567, 1067-1076 (2002).

19. Gögüus, E., Kouveliotou, C., Woods, P. M., Thompson, C., Duncan, R. C., and Briggs, M. S., ApJ, 558, 228-236 (2001).

20. Gavriil, F. P., Kaspi, V. M., and Woods, P. M., ApJ (2003), submitted (astro-ph/0310852

21. Kothes, R., Uyaniker, B. ., and Yar, A., ApJ, 576, 169-175 (2002).

22. Gögüs, E., Woods, P. M., Kouveliotou, C., van Paradijs, J., Briggs, M. S., Duncan, R. C., and Thompson, C., ApJ, 526, L93-L96 (1999).

23. Gögüs, E., Woods, P. M., Kouveliotou, C., van Paradijs, J., Briggs, M. S., Duncan, R. C., and Thompson, C., ApJ, 532, L121-L124 (2000). 